\title{
Microcontroller Based Capacitive Mass Measuring System
}

\author{
A. Abu_Al_Aish, M. Rehman, M. Z. Abdullah and A. H. Abu Hassan \\ School of Electric and Electronic Engineering, Engineering Campus University Sains Malaysia, \\ USM, 14300 Nibong Tebal, Malaysia, amirabulaish@gmail.com
}

\begin{abstract}
The paper deals with a new design of a capacitive sensor developed for the accurate and precise measurement of mass. It employs a co-axial cylinder based capacitive sensor whose capacitance decreases linearly with mass, due to the shielding effect of the conducting shield, which moves between the stationary cylinders of the capacitor with the movement of the mass holder. An accurate capacitance to voltage converting system is used to detect the mass in terms of voltage. Linear relationship between mass and output signal is obtained with the help of microcontroller. The presented sensor has high order of linearity, flexibility in design and controllable damping. The overall size of the sensor may be reduced to very small values by this design. It is highly robust and may be attached to any complex system. For the spring, used in the system, mass sensor can measure up to $7 \mathrm{~kg}$.
\end{abstract}

Keywords: Capacitive sensor; Mass sensor; Capacitance measuring system; Microcontroller; spring.

\section{INTRODUCTION}

$M^{\prime s i n}$ ASS MEASURING systems are as old as human civilization. Generally, equal arm or unequal arm balances are used in the accurate and precise measurement of masses. Automatic measurement of mass started with the help of strain gauge load cells and it is still prevailing in most of the automatic systems. However, strain gauge is temperature sensitive and the overall deflection, in load cell, is very small. Hence, chances of temperature error are present and proper compensation is provided for precise measurement of mass [1, 2].

Capacitive sensors are increasingly becoming common because they can be built with affordable technologies, and they have high impedance which implies low power consumption [3, 4]. They have been employed in many measurement schemes in the laboratories as well as in the industrial activities. They are employed in the measurement of different physical quantities such as displacement, force, pressure, density and concentration of substances [5, 6].

This paper presents an improved version of the sensors developed by the authors $[7,8]$. They are better in design, linearity and simple in fabrication.

\section{THEORY AND DESIGN}

Accuracy, small size, low power consumption and freedom from imperfections made the capacitive sensors successful in the measurement of many physical quantities [9].The capacitive sensors usually have low capacitances and their measurement is affected by the stray capacitances and earth admittances. Fig.1(a) and (b) show a three terminal capacitor and its equivalent circuit respectively.

The capacitance between the main electrodes, $\mathrm{C}_{12}$, is the designed capacitance of the sensor and it will be free from the effects of earth admittances, $C_{g}$, if the shield is grounded. If capacitance is measured between terminals 1 and 2, $\mathrm{C}_{13}$ and $\mathrm{C}_{23}$ will appear across $\mathrm{C}_{12}$, which will provide undesirable results. Hence, the capacitance measuring circuit should be such that the effect of $\mathrm{C}_{13}$ and $\mathrm{C}_{23}$ is perfectly eliminated in the final results.

The proposed sensor consists of three cylinders, two cylinders form a concentric capacitor and the third cylinder

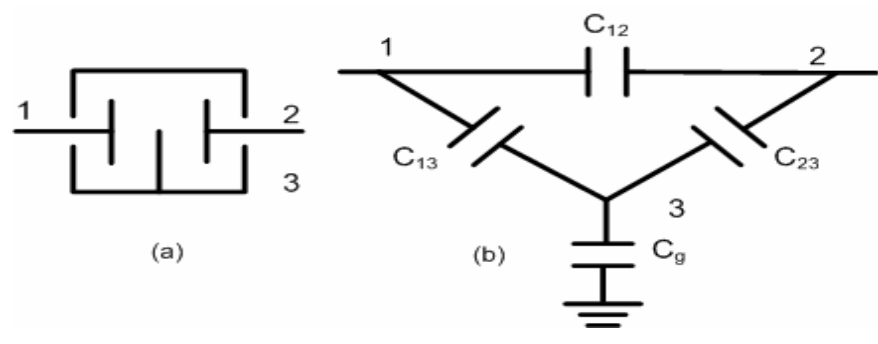

Fig.1(a) Three- Terminal Capacitor and (b) Equivalent circuit of the capacitor

acts as an electromagnetic shield. The capacitance varies between the main cylinders when a cylinder, acting as shield, moves between the main cylinders. This type of variation has two major advantages. Firstly, variation may be perfectly linear and secondly, variation in distance, between shield and electrodes, will not have any effect on the value of $C_{12}$.

Fig.2 shows a cross-sectional view of the proposed sensor. Two co-axial cylinders (1\&2) make the main capacitor. These are fixed on a Perspex base and terminals are taken out through shielded terminals. The design of the shielding cylinder (3) is different from the previous design [7]. It consists of one part without any window which decreases the size of sensor without losing any advantage. It is fixed on the mass carrying platform (4) and rests on the spring (5) which is provided to calibrate the mass. A Teflon guide (6) is provided to keep the movement of the shield vertical and in between the main cylinders (1\&2). Spring is provided with a guide (7) so that the spring may provide uniform force against the applied mass. A screw (8), with fine threads, is provided to adjust the position of the shield as well as to change the spring to cover different ranges. The whole system is contained in a metallic container (9) which provides a stable base as well as an electromagnetic shield against periodic fields. Guide of the shield (6) is provided with a hole (10) which may help in the adjustment of the damping of the system. Shielded connections are taken from the two cylinders (1\&2) and simple connection is taken from the outer container. 


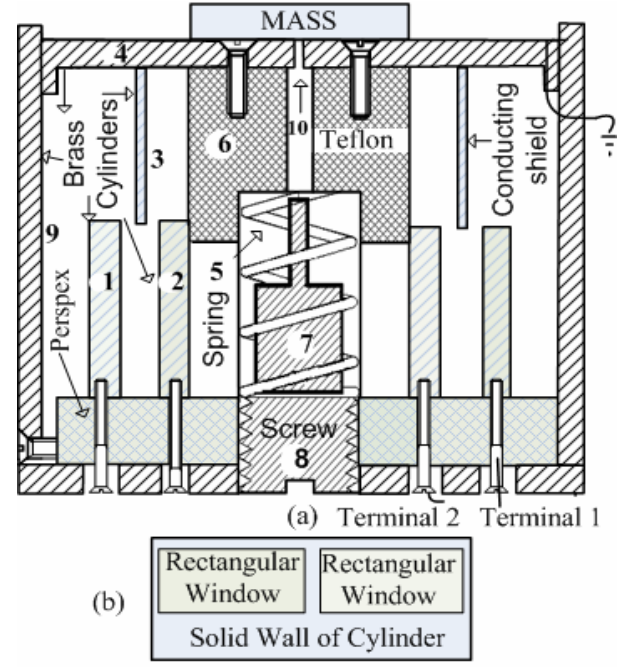

Fig.2(a) Cross- Sectional view of the mass sensor (b) Cut-Opened View of the Shield

As the mass increases, depending upon the spring constant, some downward movement of the shield takes place. The shield is designed and placed in a way that the maximum capacitance exists when no mass under measurement is added. When mass is added on the platform, the shield will move down between the two main cylindrical electrodes resulting in the linear decrease of direct capacitance $\mathrm{C}_{12}$. Relationship between mass and capacitance can be given by the following expression:

$$
C_{12}=-K M
$$

where $\mathrm{M}$ is the mass under measurement and $\mathrm{K}$ is the factor which depends upon the spring constant.

\section{Design of Electronic Measuring Circuit}

If a current is passed through an unknown capacitance, it will show a characteristic magnitude and phase shifts [9]. The phasor diagram between applied voltage and currents is depicted in Fig.3
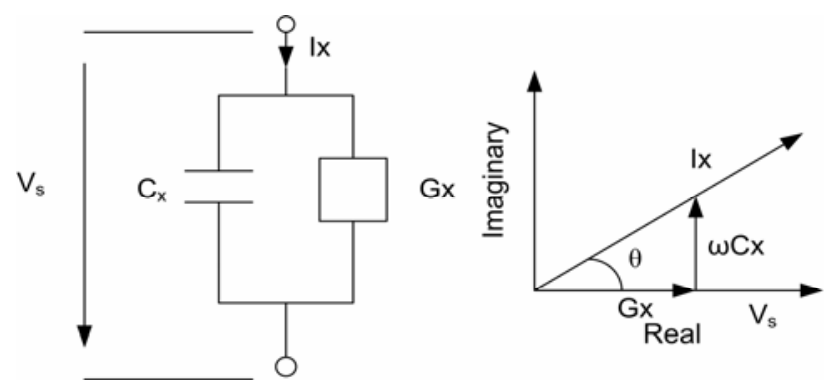

Fig.3 Equivalent circuit of a real capacitive sensor and its complex representation.

The phase shift between the excitation voltage $\left(\mathrm{V}_{\mathrm{S}}\right)$ and the resulting current $\left(\mathrm{I}_{\mathrm{X}}\right)$ will depend upon the value of the capacitance and the conductance of the capacitive sensor. If we can separate out the in-phase and quadrature components, the capacitance may be measured from the quadrature component only. The electronic circuit shown in Fig.4 can sense the direct capacitance, $\mathrm{C}_{12}$, of the sensor without the effects of $\mathrm{C}_{13}$ and $\mathrm{C}_{23}$. As shown in the figure, $\mathrm{C}_{13}$ will appear across supply voltage $\left(\mathrm{V}_{\mathrm{S}}\right)$ and will not affect the output voltage $\left(\mathrm{V}_{\mathrm{O}}\right)$ provided that output impedance of the supply is negligible in comparison with the reactance of the capacitance $\mathrm{C}_{13}$. The capacitance $\mathrm{C}_{23}$ appears across the input terminals of the operational amplifier (opamp) and hence will not affect the output as it appears between the ground and virtual ground terminal of the opamp. In this way, output of the circuit will be free from the effects of $\mathrm{C}_{13}$ and $\mathrm{C}_{23}$ [10].

Let $V_{S}$ be the supply voltage to the system, and $V_{O}$ be the output voltage of the circuit of block 1 as shown in Fig.4 which may be given by the following well known expression:

$$
V_{0}=-V_{S} R / Z_{X}
$$

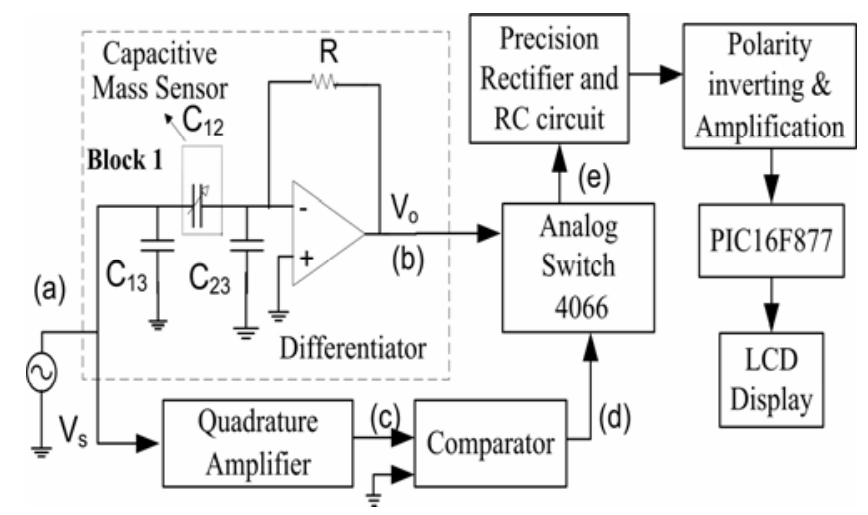

Fig.4 Schematic diagram of the capacitance measuring system

where, $\mathrm{R}$ is the feedback resistance and $\mathrm{Z}_{\mathrm{X}}$ is the impedance of the sensor composed of the parallel combination of capacitance and resistance. Substituting the values of the components of the impedance we get:

$$
V_{0}=-V_{S} R\left[\frac{1}{R_{X}}+j \omega C_{X}\right]
$$

where, $R_{X}$ and $C_{X}$ are resistance and capacitance of the sensor, connected in parallel. In (3), $\mathrm{V}_{\mathrm{O}}$ is a phasor and may be represented in terms of its in-phase $\left(\mathrm{V}_{\text {inph }}\right)$ and quadrature $\left(\mathrm{V}_{\text {quad }}\right)$ components with respect to supply voltage $\mathrm{V}_{\mathrm{S}}$ and hence can be written as:

$$
V_{o}=V_{\text {inph }}+j V_{\text {quad }}=-V_{S} R\left[\frac{1}{R_{X}}+j \omega C_{X}\right]
$$

Comparing the real and imaginary parts in (4) we get:

$$
\begin{gathered}
V_{\text {inph }}=-V_{S} \frac{R}{R_{X}} \\
V_{\text {quad }}=-V_{S} R \omega C_{X}
\end{gathered}
$$

However, considering the three terminal structure of the sensor, $\mathrm{C}_{\mathrm{X}}$ may be replaced by the direct capacitance of the sensor $\left(C_{12}\right)$ in (6) and value of $C_{12}$ may be taken from (1). We get: 


$$
V_{\text {quad }}=-V_{S} R \omega C_{12}=-K V_{S} R \omega M
$$

Equation (7) shows that the quadrature component of the output voltage, $\mathrm{V}_{\mathrm{O}}$, is directly proportional to the mass. To measure the quadrature component of the output voltage, analog switch is employed. The output of the circuit is connected to an analog switch (4066) and corresponding control signal is obtained after passing the supply voltage, $\mathrm{V}_{\mathrm{S}}$, through the $90^{\circ}$ phase shifter (unity gain) and analog comparator. Different waveforms of the input, output and control signals of the system are shown in Fig.5.

(a)

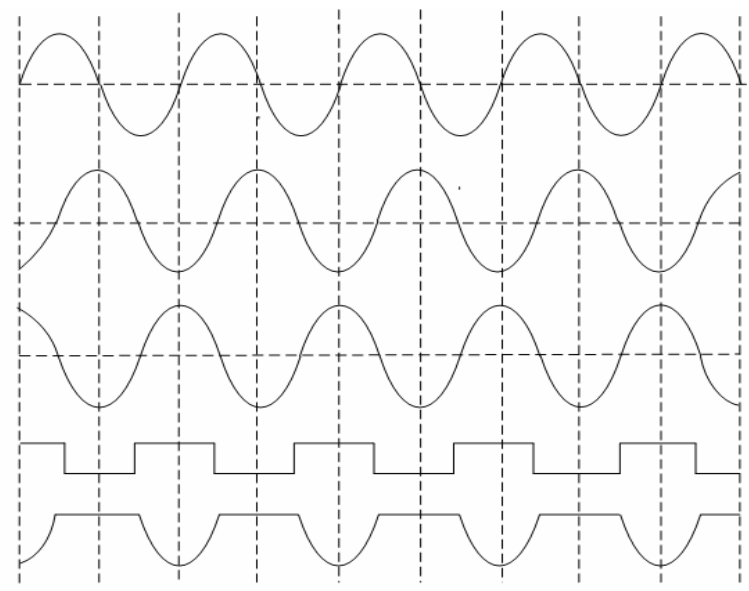

Fig.5 Waveforms of different signals (a) Waveform of supply voltage, Vs. (b) Waveform of the output voltage, Vo. (c) Waveform of the output voltage of the quadrature amplifier (gain=1) (d) Waveform of control signal to obtain quadrature component. (e) Waveform of the quadrature component when $\mathrm{ZX}=-\mathrm{jXc}$.

\section{EXPERIMENTAL METHODS AND RESUlts}

To interface the quadrature component with the microcontroller, it is necessary to get its average value as well as to invert and amplify it. For this purpose, a precision rectifier and RC circuit are used to get the average value of the quadrature component and an inverter is used to amplify as well as to change the polarity of the signal. A microcontroller is involved in the design to give the measurement system the properties of intelligence, linearity, low-cost, reliability, accuracy and adaptability. For this purpose, a microcontroller (PIC16F877) is employed. Initially, it converts the analog signal to digital form through the 10 bit internal analog to digital converter (ADC). The digital signal is calibrated, linearized and processed with the help of a designed program and the result displayed on the LCD display in terms of mass. The steps of calibration and linearization are as follows:

- Initially the system is calibrated with the help of standard mass. At zero mass, the input signal is read and stored in the memory of the system and simultaneously the output of the system is displayed as zero value.

- When the standard mass is placed on the platform, a new value of the output is obtained. It is subtracted from the initial stored value and the result is stored in another location.

- The abovementioned process is repeated for each new value of the standard mass till the complete range is covered.
- The equation of the best linearized fitting curve, between the mass and stored values, is obtained and stored in the memory and utilized in displaying the mass under measurement.

The proposed sensor is fabricated for a total movement of the upper platform (as well as of shield between the two cylinders) of the order of $1.6 \mathrm{~cm}$. The maximum mass measured with the device is $7.0 \mathrm{~kg}$ in steps of $7.14 \%$ of full scale. The results are appreciably linear with $\mathrm{R}^{2}$ (correlation coefficient) equal to 0.999 as illustrated in Fig.6.

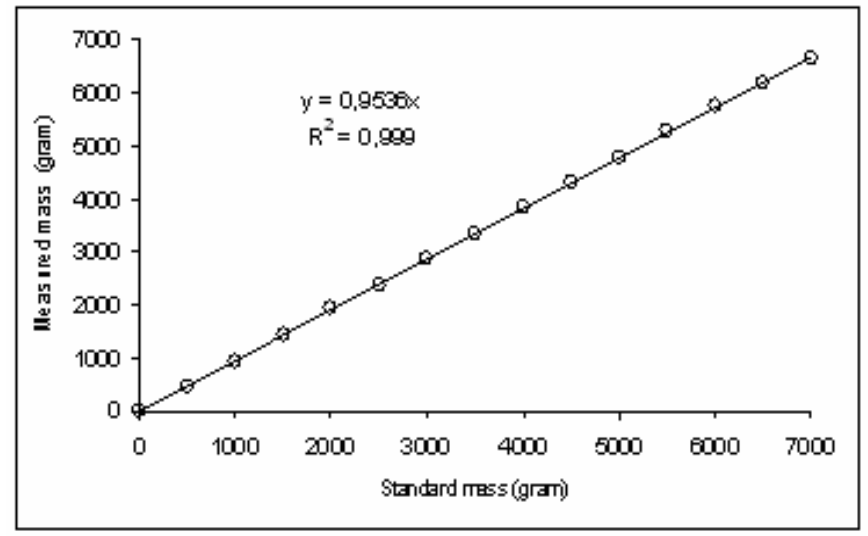

Fig.6 Relationship between measured mass and standard mass

Fig.7 shows the percentage error of the system, calculated from the displayed value and standard mass. The maximum and minimum error obtained is $\pm 0.65 \%$ and $\pm 0.02 \%$ respectively. It appears from the distribution of the error around zero value that the percentage error is nearly random and may be due to the limitations of the fabrication which can be reduced to small values with good order of fabrication.

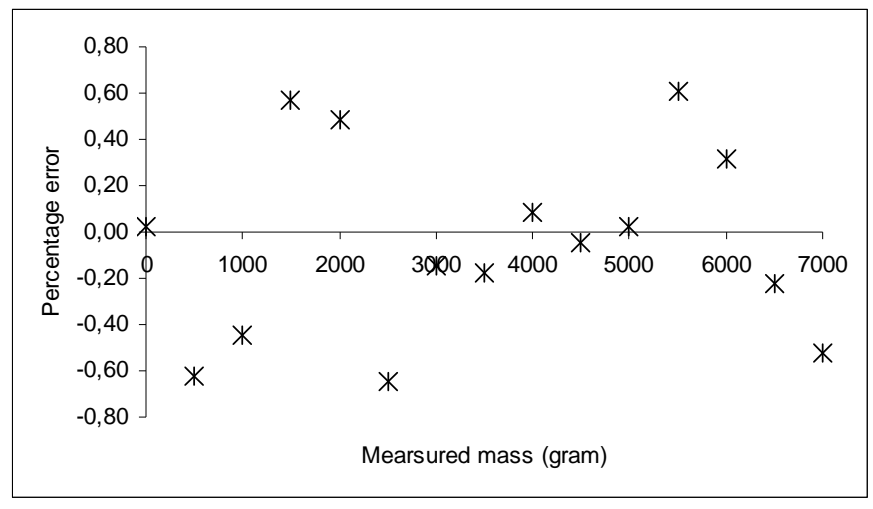

Fig.7 Percentage error of the measured mass

\section{CONCLUSION}

A linear, intelligent and robust capacitive mass measuring system with simple design is developed. It is highly flexible in design and single sensor, with slight modifications, can measure large values of masses with good level of accuracy. Its accuracy and linearity depends upon the quality of the spring and machining of the two stationary cylinders. It has all the advantages of the previous design together with the smaller size having simple shielding system. 


\section{ACKNOWLEDGMENT}

The authors would like to thank University Sains Malaysia for providing the Short term grant No. 304/PELECT/6035171.

\section{REFERENCES}

[1] Athani, V.V., Vaswani, R. (1990). PC based electronic weighing system. In Proc. of IEEE Industry Applications Society Annual Meeting. Vol. 2, 1856-1861.

[2] Norton, N.H. (1989). Handbook of Transducer. New Jersey: Prentice Hall, Inc. (72, 190-199).

[3] Marioli, D., Sardini, E., Taroni, A. (1991). Measurement of small capacitance variations. IEEE Trans. Instrum. Meas., 40 (2), 426-428.

[4] Huang, S.M., Stott, A.L., Green, R.G., Beck, M.S. (1988). Electronic transducers for industrial measurement of low value capacitance. J. Phys. E: Sci. Instum., 21, 242-280.
[5] Reverter, F., Gasulla, M., Pallis-Areny, R. (2004). A low-cost microcontroller interface for low-value capacitive sensors. Instrum. Meas., 3, 1771-1775.

[6] Baxler, L.K. (1997). Capacitive Sensors Design and Application. New York: IEEE Press.

[7] Abu_Al_Aish, A., Rehman, M. (2009). Development of a capacitive mass measuring system. Sensor. Actuator. Phys., 151 (2), 113-117.

[8] Abu_Al_Aish, A., Rehman, M., Abu Hassan, A.H., Arshad, M.R. (2009). Development of an intelligent capacitive mass sensor based on Co-axial cylindrical capacitor. Sensor. Transducer. J., 105 (6), 1-9.

[9] Kolle, C., O’Leary, P. (1998). Low-cost, high-precision measurement system for capacitive sensors. Meas. Sci. Technol., 9, 510-517.

[10] Rehman, M., Abu_Al_Aish, A. (2007). Simulation, analysis and design of an active bridge for sensor capacitance measurement, using MATLAB. In International Conference on Modeling and Simulation, 959-962.

Received October 15, 2009. Accepted February 2, 2010. 